\title{
Challenges of Difficult Airway in Treated Cancer Patient for High-risk Coronary Artery Bypass Graft Surgery
}

Rupinder K Kaiche ${ }^{1}$, Rahul A Kaiche ${ }^{2}$

\begin{abstract}
Aims and objectives: To highlight the need to formulate a difficult airway pathway for post-cancer treated patients posted for high-risk cardiac surgery. Airway protection is mandatory for surgery. Cancer patients, especially oral, are difficult airway candidates as are cardiac patients. Cancer patients pose anatomical challenges, while cardiac patients pose physiological and/or anatomical challenges. However, when a cancer-treated patient comes for cardiac surgery with compromised cardiac status, the risk and complication probability increases. With cardiac disease and cancer being interrelated and incidence rising with newer treatment modalities, cases are varied requiring impromptu innovation. We present a case of post-surgery, chemo, and radiotherapy oral cancer posted for coronary artery bypass graft (CABG) with left main disease and low ejection fraction. Keywords: CABG, Cancer, Difficult airway, Fiberoptic intubation.

Research and Innovation in Anesthesia (2021): 10.5005/jp-journals-10049-0102
\end{abstract}

\section{INTRODUCTION}

Cardiac disease and cancer represent two major causes of morbidity and mortality. Oral cancer is the most common cancer among males in India related to tobacco and gutka chewing, ${ }^{1}$ which also contribute to cardiac disease. With advances in treatment modalities, patients come for surgery at various stages of either disease and treatment process, posing challenges of disease and drugs to the anesthesiologist. The difficult airway is anticipated in cancer patients due to altered anatomy, surgery, radiation fibrosis, and physiological constraints in cardiac surgery patients. Difficult laryngoscopy exerts some hemodynamic responses which can be disastrous to a compromised heart especially in left main disease, its equivalent, low EF. ${ }^{2}$ We present a case of oral cancer treated with surgery followed by chemotherapy and radiotherapy for coronary artery bypass graft $(C A B G)$ with left main disease and low ejection fraction.

\section{Case Description}

A 55-year-old man was admitted with angina, ECG showing anterio-inferior MI. He was shifted to the cath lab on inotropes for angiography, which revealed LM-90\%, LAD-70\%, D1-90\%, LCX-70\%, and proximal RCA-70\%. Echo showed global hypokinesia with grade II diastolic dysfunction, EF-32\%. Advised CABG.

He was short-statured, moderately built with a history of HTN for 2 years. He was operated on for Ca buccal mucosa followed by chemotherapy and radiotherapy. The last cycle finished 6 months ago. He complained of DOE grade III and easy fatigability. He had off and on cough, no fever, no orthopnea. O/E restricted mouth opening with less than a finger interincisor gap. Tongue protrusion was not possible. Hence, awake fiberoptic intubation was planned. All pre-op investigations were done (Fig. 1).

On the day of surgery, 16-G IV cannula, right IJV, and right radial artery cannulated under local anesthesia. Difficult airway cart kept ready with consent for tracheostomy. Patient sedated with midazolam-fentanyl and nasal intubation attempted using 7.0 cuffed endotracheal tube with heart rate- $68 /$ minute, BP- $110 / 68 \mathrm{~mm}$ $\mathrm{Hg}, \mathrm{SpO}_{2}-98 \%$, and nikoran infusion, after preparing nasal and oral
'Department of Cardiac Anesthesia, Ashoka Medicover Hospitals, Nashik, Maharashtra, India

${ }^{2}$ Department of Cardiac Surgery, Ashoka Medicover Hospitals, Nashik, Maharashtra, India

Corresponding Author: Rupinder K Kaiche, Department of Cardiac Anesthesia, Ashoka Medicover Hospitals, Nashik, Maharashtra, India, Phone: +91 9370438083, e-mail: rrkaur8@gmail.com

How to cite this article: Kaiche RK, Kaiche RA. Challenges of Difficult Airway in Treated Cancer Patient for High-risk Coronary Artery Bypass Graft Surgery. Res Inno in Anesth 2021;6(1):21-22.

Source of support: Nil

Conflict of interest: None

cavity with a local anesthetic. However, on approaching the cords when sprayed, coughing raised HR-110/minute and BP-160/110 mm $\mathrm{Hg}, \mathrm{SpO}_{2}-96 \%$, stabilized with retraction of scope and fentanyl, propofol, and NTG. Procedure reattempted with success confirmed

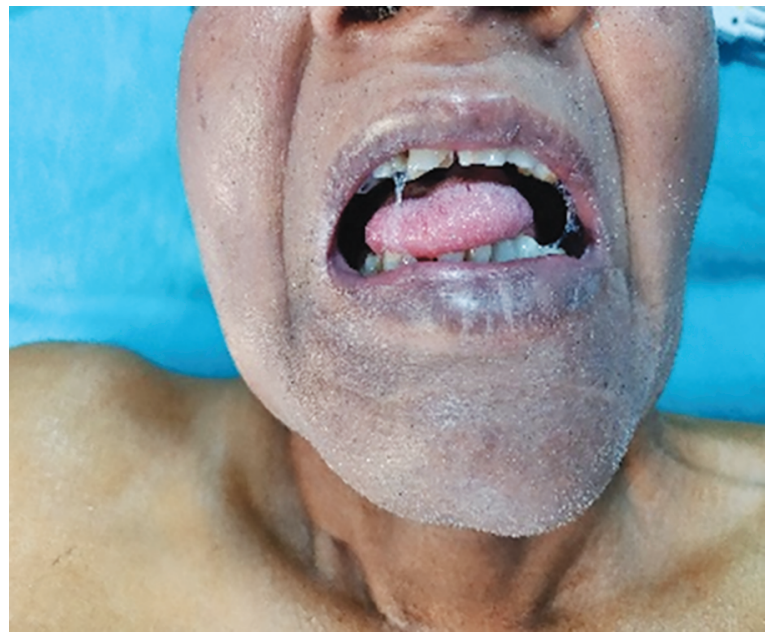

Fig. 1: Restricted mouth opening

(c) The Author(s). 2021 Open Access This article is distributed under the terms of the Creative Commons Attribution 4.0 International License (https:// creativecommons.org/licenses/by-nc/4.0/), which permits unrestricted use, distribution, and non-commercial reproduction in any medium, provided you give appropriate credit to the original author(s) and the source, provide a link to the Creative Commons license, and indicate if changes were made. The Creative Commons Public Domain Dedication waiver (http://creativecommons.org/publicdomain/zero/1.0/) applies to the data made available in this article, unless otherwise stated. 


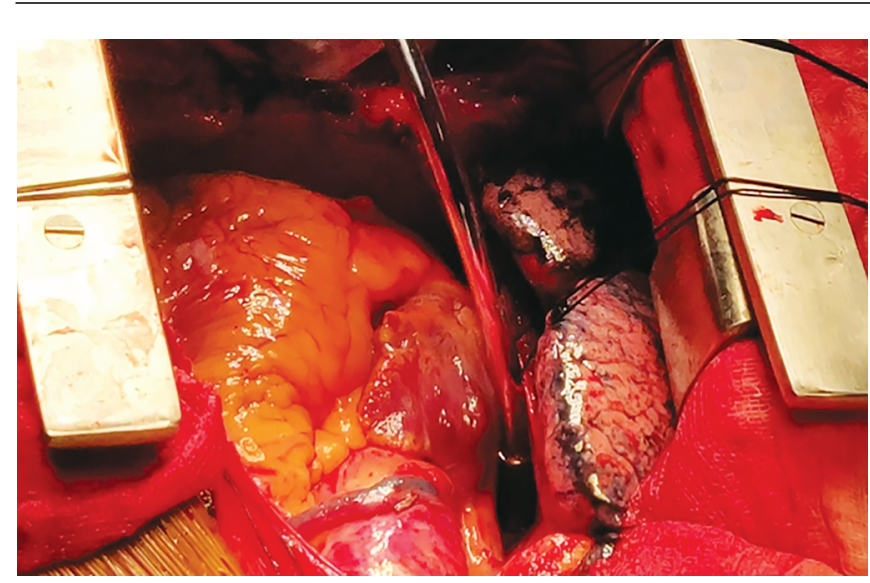

Fig. 2: Heart shows aorta-SVG-OM graft

using $\mathrm{EtCO}_{2}$ and auscultation. Propofol, midazolam-fentanylatracurium infusion, sevoflurane started to maintain BIS: 40-60. Offpump CABG was done using Octopus3 tissue stabilizer, Medtronic USA, with LIMA-LAD, Aorta-SVG-diagonal, and Aorta-SVG-OM grafts. ACT maintained between 250 seconds and 300 seconds using heparin, reversed with protamin at end of surgery. Shifted to ICU with stable hemodynamics on inotropes and glycemic control using insulin infusion (Fig 2).

\section{Extubation}

Extubation was planned after confirming hemodynamic stability, decreasing hourly drain output, urine output $>1 \mathrm{~mL} / \mathrm{kg}$, alert with respiratory rate $<20 /$ minute, tidal volume $>400 \mathrm{~mL}$, and $\mathrm{SpO}_{2}>95$. The patient was upright with a good muscle tone and cough reflex. He was closely observed for any signs of respiratory distress.

After 48 hours in ICU, shifted to the ward and discharged on day 7 with physiotherapy guidance.

\section{Discussion}

Difficult airway, commonly encountered in patients for cardiac surgery, can be anatomical or physiological. It is imperative to consider all cardiac patients as potential Difficult airway since low cardiac output syndromes and acute heart failure ensue abruptly. The standard hemodynamic response after laryngoscopy and intubation was observed as tachycardia and hypertension occur due to stimuli to oropharyngeal structures resulting in sympathetic fibers of cervical plexus getting stimulated. In addition, the spinal cord produces a diffuse autonomic response that releases noradrenaline, adrenaline, and renin, producing a pronounced response by sympathetic hyperactivity presenting noradrenaline circulating levels 2-3 times higher. ${ }^{3}$ The heart rate increases and EF decreases in the first minute post-intubation increasing the oxygen myocardial consumption $25-54 \%$ which along with the debit of coronary sinus oxygen produces a failure in coronary selfregulation. ${ }^{4}$ Another cause of D.A is cancer-treated anatomical distortion post-surgery, radiation therapy especially oral and thoracic cancers. Chemo and radiotherapy-treated patients also develop CAD. ${ }^{5}$ Cancer survivors are prone to develop heart failure due to cardiotoxic antineoplastic agents and clustering of cardiovascular risk factors in oncology patients. Conversely, studies

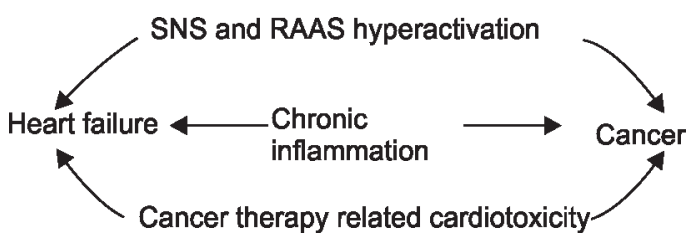

Fig. 3: Mechanism underlying the bidirectional relationship between heart failure and cancer. SNS, sympathetic nervous system; RAAS, reninangiotensin-aldosterone system

have reported an increased risk of incident cancer in heart failure patients. ${ }^{6-9}$ The common pathological state of chronic low-grade inflammation predisposes to both (Fig. 3). ${ }^{10}$

\section{ConCLUSION}

Airway management can be very challenging in cancer patients often requiring manipulation. However, if the patient is undergoing cardiac surgery the hemodynamic response to manipulation can cause serious cardiac decompensation. Difficult airway management protocols should take cognizance of this fact. Despite a detailed explanation of the procedure with the patient, anxiolysis, moderate sedation, and coronary dilators, we still experienced hemodynamic fluctuations needing a second attempt at intubation. Regular skill and equipment upgradation is warranted for high-risk cases to decrease complications.

\section{References}

1. Yusuf SW, Howell RM, Gomez D, et al. Radiation-related heart and vascular disease. Future Oncol 2015;11(14):2067-2076. DOI: 10.2217/ fon.15.129.PMID:26198837.

2. Veena N, Pradeep V. Incidences and predictors of difficult laryngoscopy in patients undergoing coronary artery bypass grafting. J Evid Based Med Healthc 2018;5(49):3363-3368. DOI: 10.18410/jebmh/2018/685.

3. Bruder N, Ortega D, Granthil C. Consequences and prevention methods of hemodynamic changes during laryngoscopy and intra tracheal intubation. Ann Fr Anesth Reanim 1992;11(1):57-71. DOI: 10.1016/s0750-7658(05)80321-1.

4. Martinez Hurtado E, Sanchez Merchante M. Hemodynamic response to endotracheal intubation using C-trach assembly and direct laryngoscopy. Saudi J Anaesth 2016;10(4):485-486. DOI: 10.4103/1658-354X.177335.

5. Wu W, Masri A, Popovic ZB, et al. Long term survival of patients with radiation heart disease undergoing cardiac surgery: a cohort study. Circulation 2013;127(14):1476-1485. DOI: 10.1161/ CIRCULATIONAHA.113.001435.

6. Banke A, Schou M, Videbaek L, et al. Incidence of cancer in patients with chronic heart failure: a long-term follow-up study. Eur J Heart Fail 2016;18(3):260-266. DOI: 10.1002/ejhf.472.

7. Hasin T, Gerber Y, Mc Nallan SM, et al. Patients with heart failure have an increased risk of incident cancer. I Am Coll Cardiol 2013;62(10):881886. DOI: 10.1016/j.jacc.2013.04.088.

8. Hasin T, Gerber Y, Weston SA, et al. Heart failure after myocardial infarction is associated with increased risk of cancer. J Am Coll Cardiol 2016;68(3):265-271. DOI: 10.1016/j.jacc.2016.04.053.

9. Sakamoto M, Hasegawa T, Asakura M, et al. Does the pathophysiology of heart failure prime the incidence of cancer? Hypertens Res 2017;40(9):831-836. DOI: 10.1038/hr.2017.45.

10. Bertero E, Canepa M, Maack C, et al. Linking heart failure to cancer. Circulation 2018;138(7):735-742. DOI: 10.1161/ CIRCULATIONAHA.118.033603. 